\title{
Laboratory Studies of Surface Waves Using a Laser Doppler Vibrometer
}

\author{
Koichi Hayashi ${ }^{1)}$ and Osamu Nishizawa ${ }^{2)}$ \\ 1) OYO Corporation, Tsukuba, Ibaraki, Japan \\ 2) Geological Survey of Japan, Tsukuba, Ibaraki, Japan
}

\begin{abstract}
We have performed physical modeling of surface waves using a laser Doppler vibrometer (LDV). Surface wave methods have been applied to both engineering and earth science problems to image S-wave velocity of the Earth. In order to develop the surface wave methods, numerical and physical modelings of surface waves are important. The LDV converts a particle velocity of media to the Doppler shift frequency, and enables us to measure very precise ultrasonic waves. A piezoelectric transducer (PZT) was used as a source of elastic waves. $50 \mathrm{KHz}$ to $500 \mathrm{KHz}$ elastic waves were observed by using the LDV and PZT. We have measured surface waves propagating a homogeneous half space models and horizontal two-layer models using the LDV and PZT. Theoretical characters of surface waves, such as Rayleigh wave dispersion, have been clearly observed by the physical modeling.
\end{abstract}

\section{Introduction}

In the early 1980 s, a spectral analysis of surface waves (so called SASW) was introduced. Park et al. (1999a, 1999b) have improved the SASW to the multi-channel analysis of surface waves (MASW) and Xia et al. (1999a) generated two-dimensional S-wave velocity map by the MASW. The surface wave methods, such as the SASW or MASW, can estimate S-wave velocity models that are extremely valuable in engineering work. Therefore, the surface wave methods have been increasingly used in various civil engineering and environmental investigations.

The surface wave methods for near-surface problems are relatively new technique. Therefore, the methods have several important difficulties that should be solved as follows. The methods use the dispersive character of Rayleigh waves, especially fundamental mode of surface waves. Body waves and higher mode surface waves can contaminate the data in the surface wave methods, and the separation of the fundamental mode surface waves from those unfavorable waves may be one of the difficulties in the surface wave method analysis. The surface wave method analysis relies on a one-dimensional analysis of dispersion curves (Xia et al., 1999a, $1999 \mathrm{~b}$ ). Therefore, the analysis of dispersion curves may have some difficulties in two- and three-dimensional velocity models. The number of theoretical studies about surface wave propagation in the heterogeneous near-surface region is relatively small. For example, only few attempt have so far been made at the effect of two- and three-dimensional models on the method.

In order to improve the accuracy and reliability of the surface wave methods, we have to understand fundamental characters of surface waves. Both numerical modeling, such as finite-difference methods, and physical modeling can be valuable tools for this objective. In this paper, we will perform physical modeling by the LDV in order to the fundamental characters in the surface waves, such as Rayleigh wave dispersion, in one-dimensional models.

\section{Experiment}

A light beam reflected from a moving object has a Doppler shift $f_{D}$ :

$$
f_{D}=2 V / \lambda
$$


where $V$ and $\lambda$ denote the velocity of object along the beam and the wavelength of light respectively. We employed a LDV LV-1300(ONO sokki Inc.), which is used for measuring vibration of factory product. The Doppler shifted frequency $f_{D}$ is converted to a voltage signal that indicates actual vibrations. Since the velocity is directly converted to the frequency shift, there is neither the mechanical nor the electrical coupling between the material's surface vibration and the observed signal. Nishizawa et al.(1997) used LDV for measuring seismic waves at the surface of the rock prisms and have proved it can be used as a precise receiver for ultrasonic waves $(300 \mathrm{KHz}$ to $2 \mathrm{MHz}$ ) generated by the PZT.

Figure 1 is a schematic diagram of the measurement system. The detail of the system is described in Nishizawa et al.(1997). A source is a 5-mm PZT having a $2 \mathrm{MHz}$ characteristic frequency. We used driving pulses that consists of a single-cycle sine wave with 100 to $500 \mathrm{KHz}$ frequency and $100 \mathrm{~V}$ peak to peak amplitude. A thin reflection sheet is glued to the model surface to enhance the reflection beam.

\section{Homogeneous models}

In the first example, homogeneous half space models are used. Square prisms made of acrylic and gabbro were used as homogeneous models. The size of model is $300 * 300 * 90 \mathrm{~mm}$. Source-receiver configuration is shown in Figure 2. Waveforms were collected the surface of the models with $4 \mathrm{~mm}$ distance interval. The first receiver was placed $4 \mathrm{~mm}$ apart from the source. Since a source and receivers are placed on the surface of homogeneous models, this example can be considered as the Lamb's problem.

Figure 3 shows the vertical component of particle velocities and its power spectrum for the acrylic model. 100 and $250 \mathrm{KHz}$ single-cycle sine waves were used as driving pulses. Small amplitude first arrival with the velocity of $2.7 \mathrm{~km} / \mathrm{s}$ and large amplitude later phase with the velocity of $1.25 \mathrm{~km} / \mathrm{s}$ are clearly observed. Figure 4 shows the vertical component of particle velocities and its power spectrum for the gabbro model. 100 and $250 \mathrm{KHz}$ single-cycle sine waves were used as driving pulses. Large amplitude later phase with the velocity of $3.2 \mathrm{~km} / \mathrm{s}$ is clearly observed. First arrival cannot be clearly seen from Figure 4 due to low gain. The velocity for the first arrival seems to be $5.6 \mathrm{~km} / \mathrm{s}$ from a figure with high gain. In the waveforms for the $250 \mathrm{KHz}$ pulse, clear later phase with a hyperbolic curve can be observed beyond $100 \mu \mathrm{s}$. This phase seems to be reflected surface waves (Rayleigh waves) from the edge of the model. Figure 5 shows the phase velocity - frequency images of waveform data converted through the multi-channel analysis of surface waves (Park et al., 1999a).

From these figures, waveforms for this example can be characterized as follows. Waveforms consist of small amplitude first arrival and large amplitude later phase. It is obvious that the former is direct P-waves and the latter is surface waves (Rayleigh waves). The amplitude of Rayleigh waves is much larger than that of direct P-waves. Both P-waves and Rayleigh waves have little dispersion. These characters agree with the analytical solution of Lamb's problem (Saito, 1993). We can be fairly certain that the measurement system with the PZT and the LDV has generated and measured correct surface waves.

\section{Two-layer models}

In the next example, horizontal two-layer models are used. The models consist of acrylic as a low-velocity first layer and mortar as a high-velocity second layer. Two models are used in the experiment and the thickness of first layer is $5 \mathrm{~mm}$ and $10 \mathrm{~mm}$ respectively. Source-receiver configuration is the identical with the first example. 100 and $500 \mathrm{KHz}$ single-cycle sine waves 
were used as driving pulses.

Figure 6 shows the vertical component of particle velocities and its phase velocity image for a model with $5 \mathrm{~mm}$ thickness first layer. In the case of a $100 \mathrm{KHz}$ pulse, two clear later phases are observed. The phase velocity for the faster one is about $1.9 \mathrm{~km} / \mathrm{s}$ and the slower one is about $1.25 \mathrm{~km} / \mathrm{s}$. These two later phases that can be considered as surface waves, compose a clear dispersion curve on the phase velocity image. We can say with fair certainty that the curve is the fundamental mode of Rayleigh wave dispersion curves. In the case of a $500 \mathrm{KHz}$ pulse, waveform appearance is too complicated to identify phase velocity. However, the dispersion curves appeared on the phase velocity image is almost identical with the $100 \mathrm{KHz}$ case. It is amazing that such different waveforms have almost identical phase velocity images. This result shows the accuracy of the measurement system as well as the robustness of the phase velocity - frequency transformation method through the multi-channel analysis of surface waves.

Figure 7 shows the vertical component of particle velocities and its phase velocity image for a model with $10 \mathrm{~mm}$ thickness first layer. A clear dispersion curves can be seen on the phase velocity image. However, the phase velocity for the second layer cannot be easily determined from the dispersion curves in comparison with the $5 \mathrm{~mm}$ case.

The result indicates that the velocity model is more important than the frequency contents of the source in order to image clear dispersion curves. Park et al. (1999a) shows that the resolution of phase velocity image increases as the number of traces increases to include further offset. It seems reasonable to suppose that the resolution of dispersion curves is determined by velocity models, the number of traces and the inclusion of further offset. The frequency contents of the source may have small effect on the dispersion curves as long as the data acquisition is precise.

Dispersion curves are picked as the maximum amplitude in each frequency on the phase velocity images. Picked dispersion curves are shown in Figure 8. A non-linear least square method is applied to the dispersion curves in order to obtain velocity models. The number and thickness of layer, and densities are fixed during the iteration. $\mathrm{P}$-wave velocities are linearly related to $S$-wave velocities by two constants. Therefore, only $S$-wave velocities are unknown in the inversion. The inverted velocity models (S-wave velocity) are shown in Figure 9. Almost correct layer thickness $(5 \mathrm{~mm}$ and $10 \mathrm{~mm})$ was obtained by the inversion. The result shows the reliability of the whole process of surface wave analysis including both the computation of dispersion curves from waveforms and its inversion.

\section{Conclusions}

The physical modeling of surface waves using the LDV and PZT was performed and the fundamental characters in the surface waves were studied. The waveforms from the modeling for homogeneous half space agreed with the analytical solution of Lamb's problem. It proves that the LDV can measure and the PZT can generate the surface waves. Clear dispersion curves were obtained from the waveforms for two-layer models. It shows the accuracy of the measurement system as well as the robustness of the phase velocity - frequency transformation method. Almost correct velocity models were obtained by the inversion of the dispersion curves. We may say that the reliability of surface wave methods has been shown by this physical modeling.

\section{References}

Nishizawa, O., Satoh, T., Lei, X. and Kuwahara, Y., 1997, Laboratory studies of seismic wave propagation in inhomogeneous media using a laser Doppler vibrometer, BSSA, 87, 809-823.

Park, C. B., Miller, R. D. and Xia , J., 1999a, Multimodal analysis of high frequency surface 
waves, Proceedings of the symposium on the application of geophysics to engineering and environmental problems '99, 115-121.

Park, C. B., Miller, R. D., and Xia, J., 1999b, Multichannel analysis of surface waves, Geophysics, 64, 800-808.

Saito, M., 1993, Branch line contribution in Lamb's problem, BUTSURI-TANSA, 46, 372-380 (in Japanese).

Xia, j., Miller, R. D. and Park, C. B., 1999a, Configuration of near-surface shear-wave velocity by inverting surface wave, Proceedings of the symposium on the application of geophysics to engineering and environmental problems '99, 95-104.

Xia, j., Miller, R. D. and Park, C. B., 1999b, Estimation of near-surface shear-wave velocity by inversion of Rayleigh waves, Geophysics, 64, 691-700.

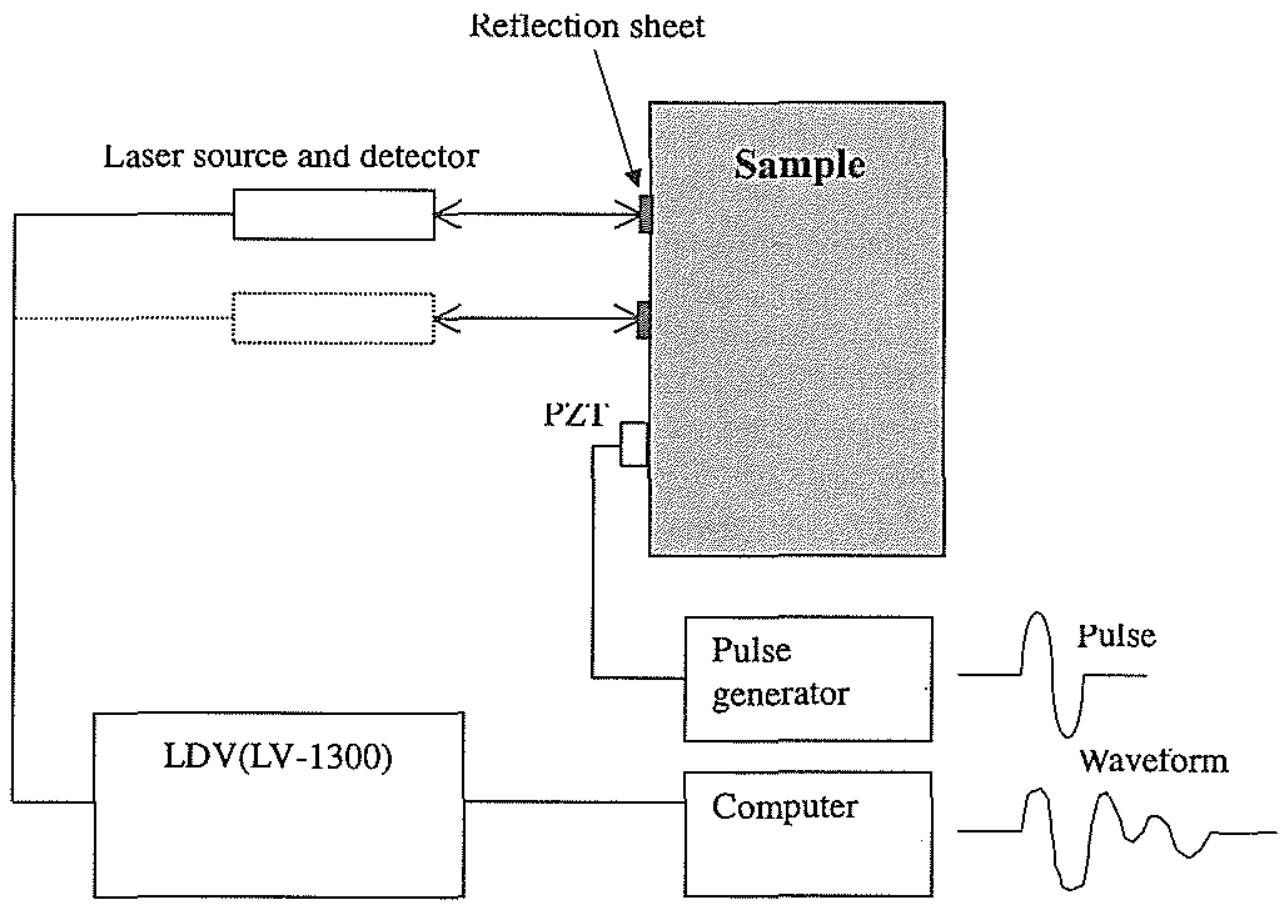

Figure 1. Schematic diagram of the measurement system using a laser Doppler vibrometer (LDV) and a piezoelectric transducer (PZT). The PZT is mounted on the surface for generating elastic waves. The laser beam illuminates the high-gain reflection sheet, which reflects the beam in an incident direction. 


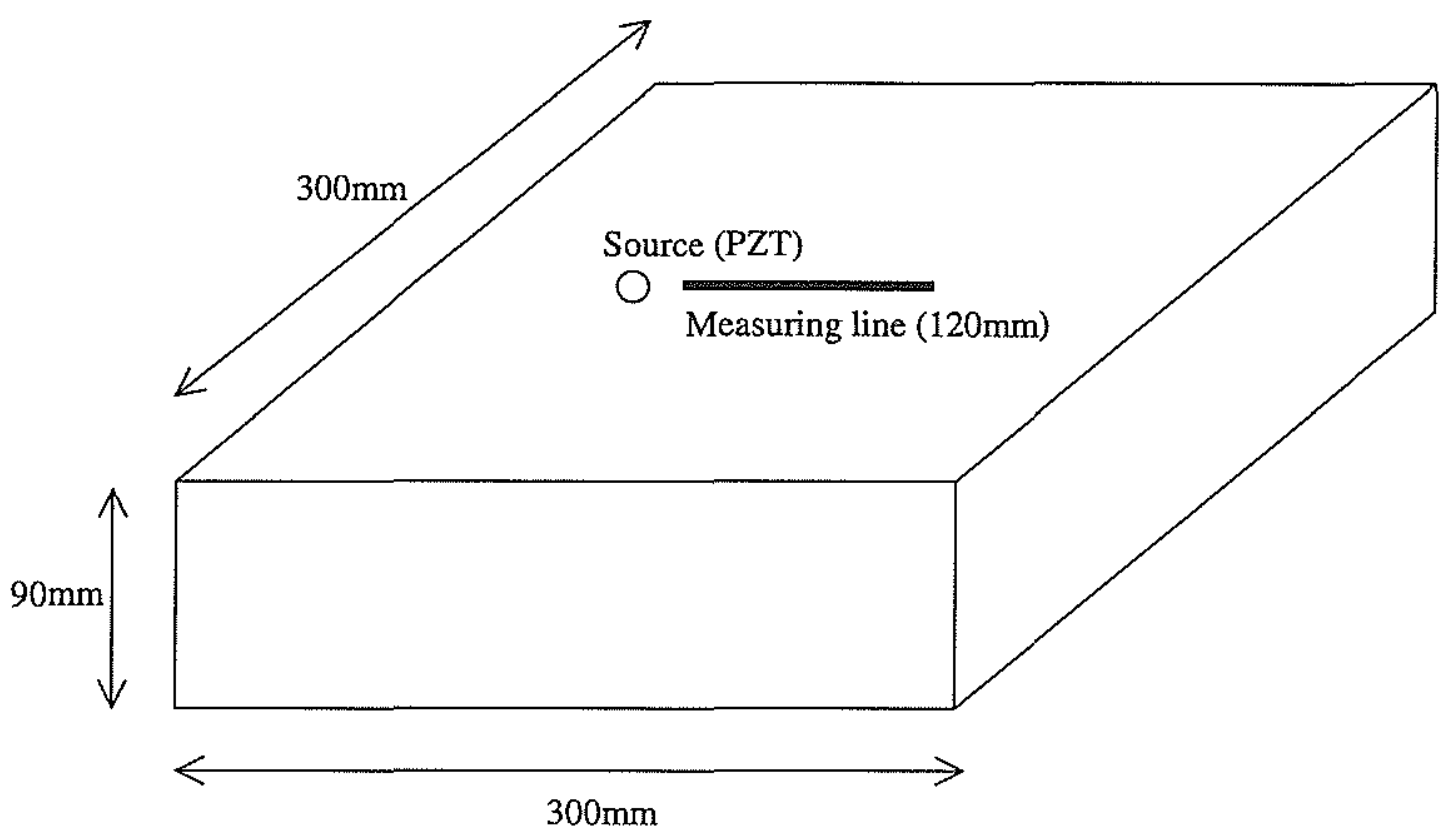

Figure 2. Source-receiver configuration used in the experiment. A source and a measuring line are placed in the middle of the models. Waveforms are collected at the surface of the models with $4 \mathrm{~m}$ distance interval.

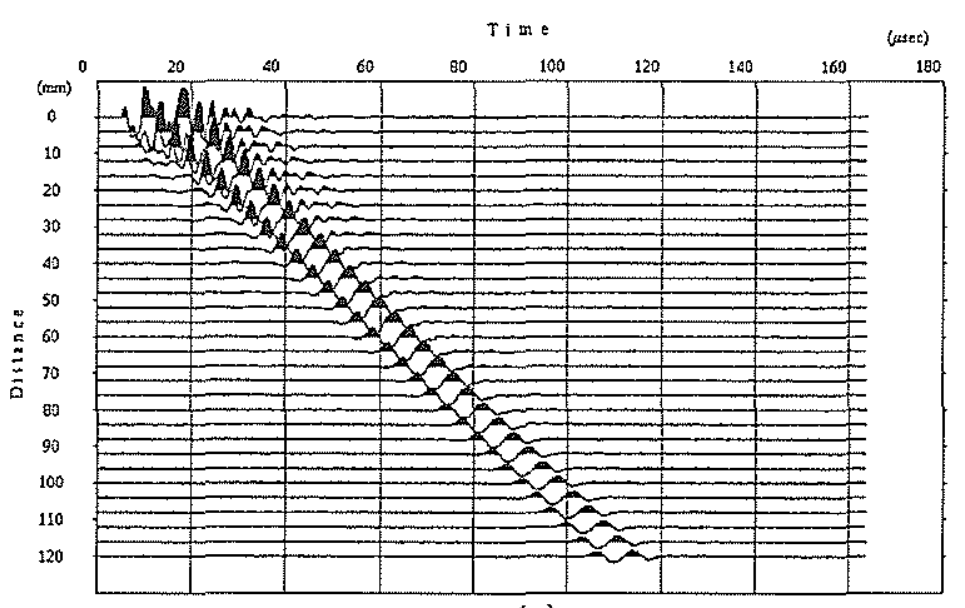

(a)

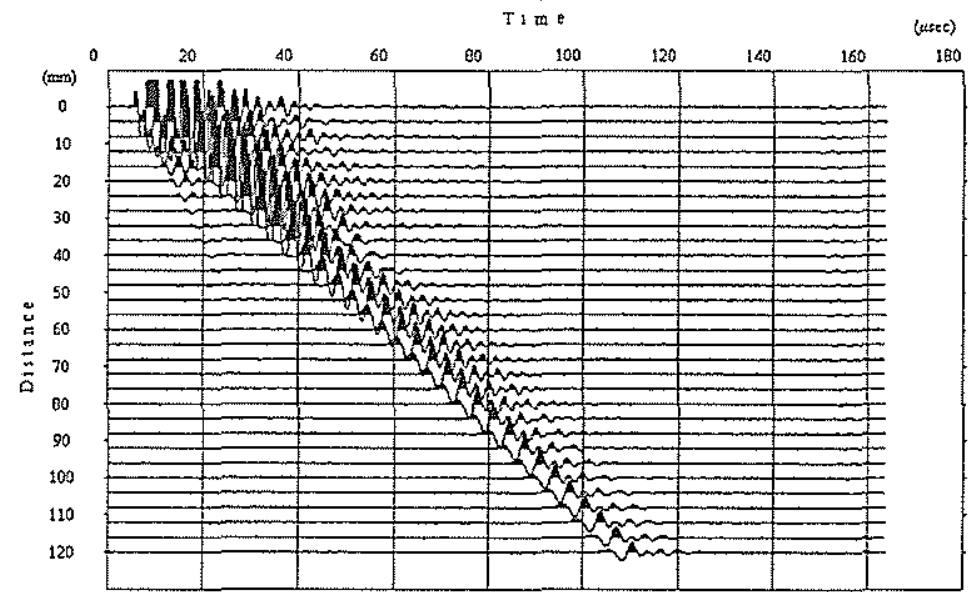

(c)

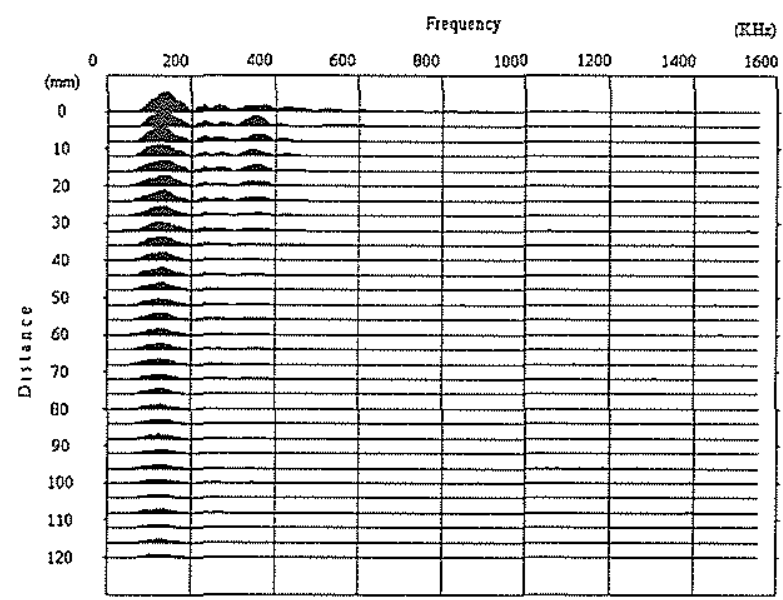

(b)

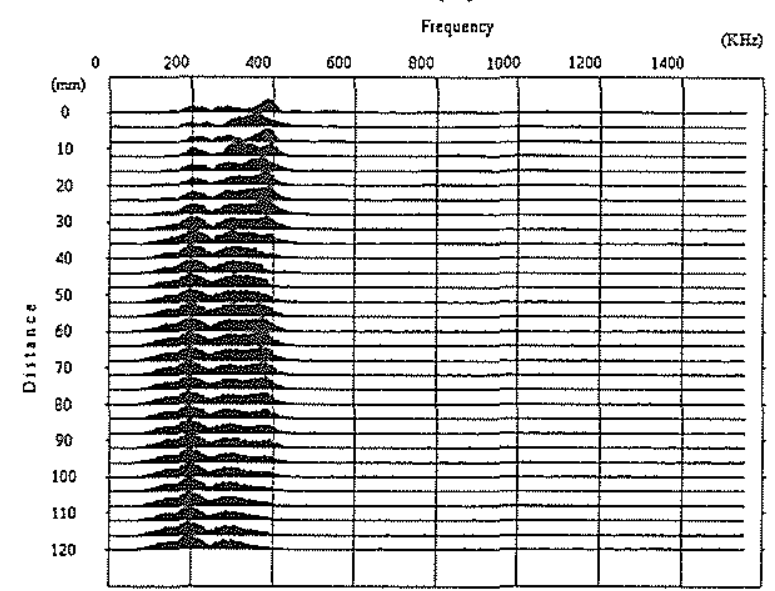

(d)

Figure 3. Vertical component of particle velocities and its power spectrum for the acrylic model. (a) Waveforms and (b) power spectrum for $100 \mathrm{KHz}$ pulse. (c) Waveforms and (d) power spectrum for $250 \mathrm{KHz}$ pulse. 


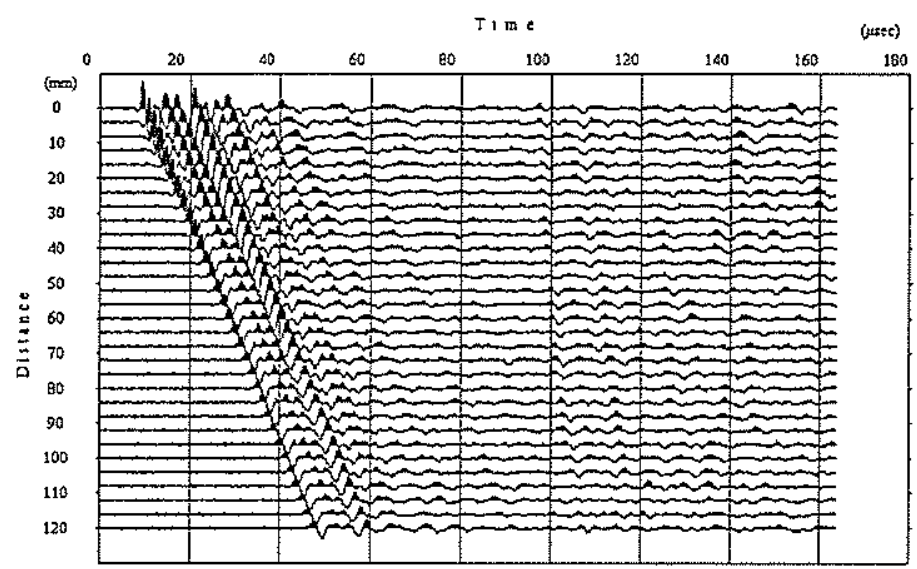

(a)

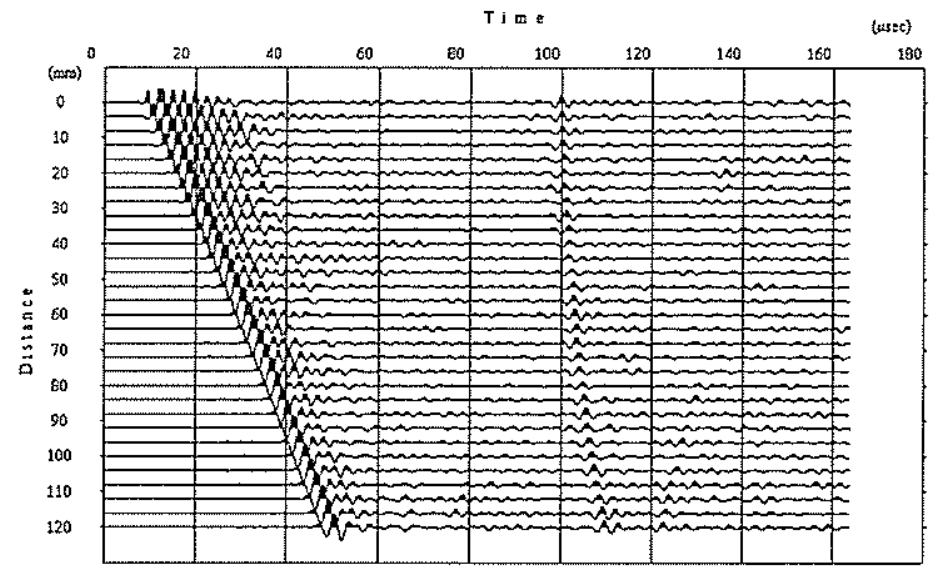

(c)

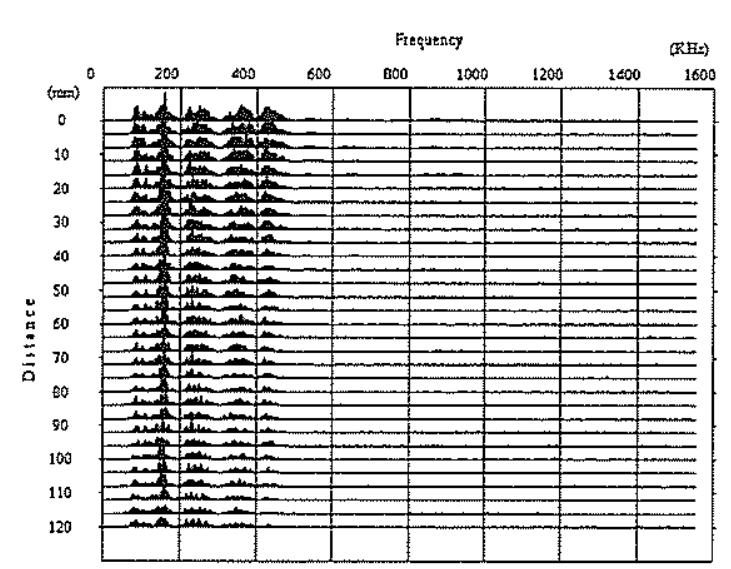

(b)

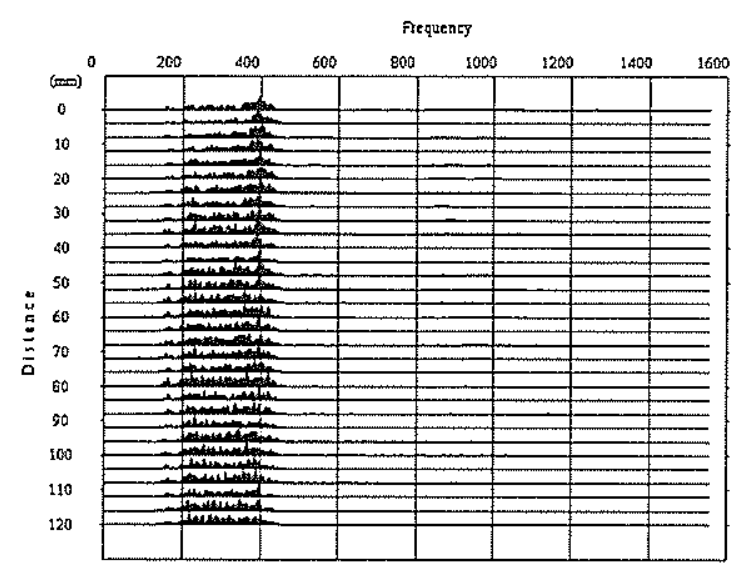

(d)

Figure 4. Vertical component of particle velocities and its power spectrum for the gabbro model. (a) Waveforms and (b) power spectrum for $100 \mathrm{KHz}$ pulse. (c) Waveforms and (d) power spectrum for $250 \mathrm{KHz}$ pulse.

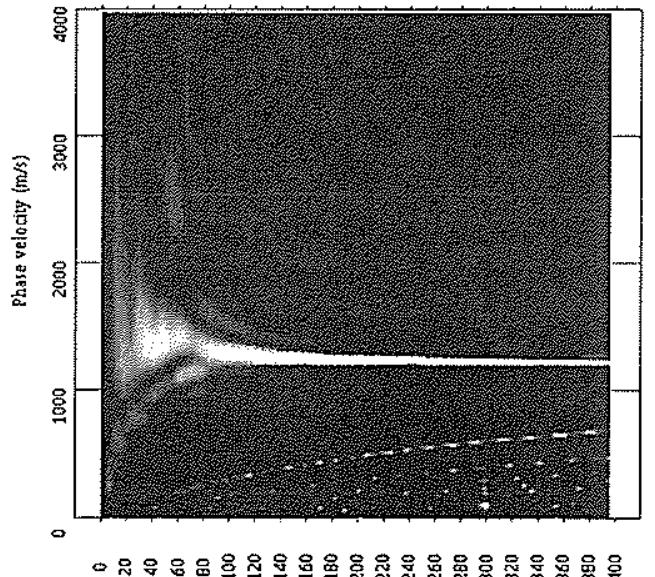
Frequtasy(KHz)

(a)

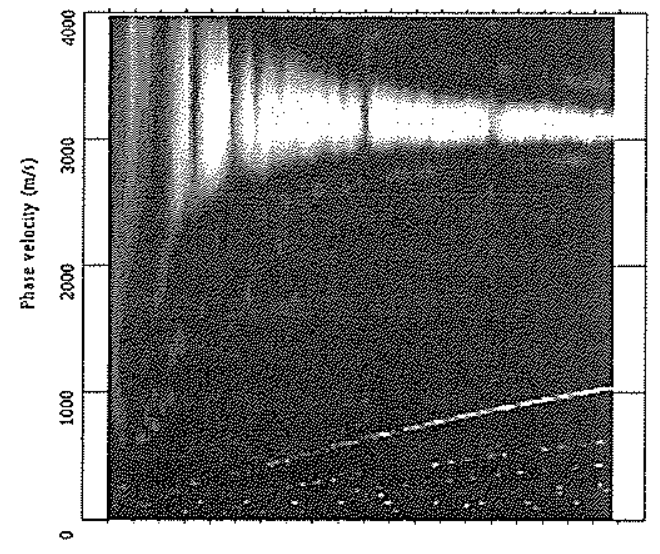

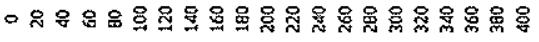
Frequenty $(\mathrm{KH} x)$

(b)

Figure 5. Phase velocity images converted through the multi-channel analysis of surface waves. (a) Waveforms for acrylic model. (b) Waveforms for gabbro model. 


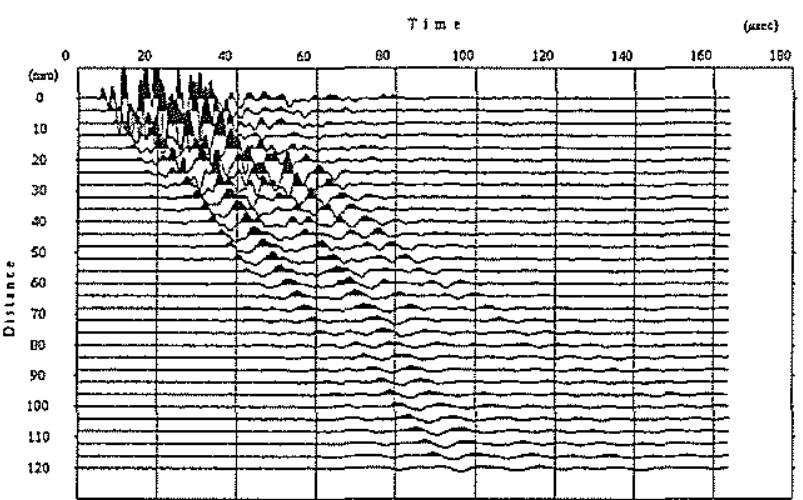

(a)

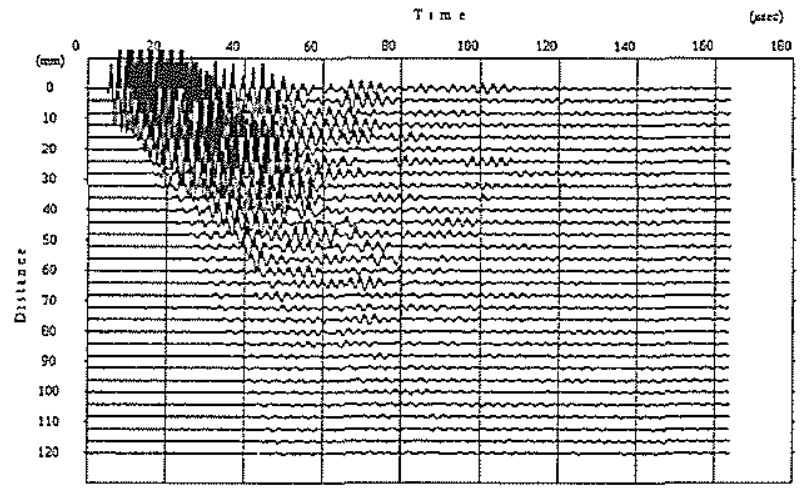

(c)

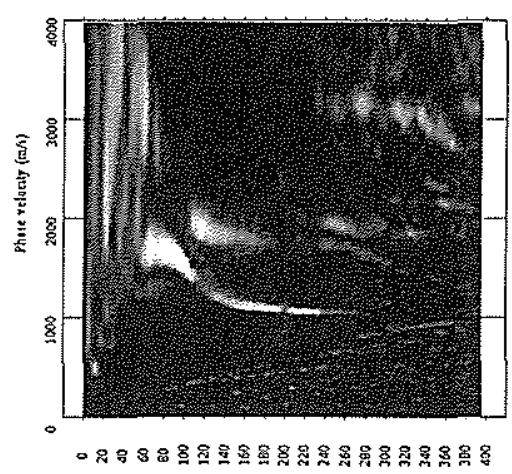

(b)

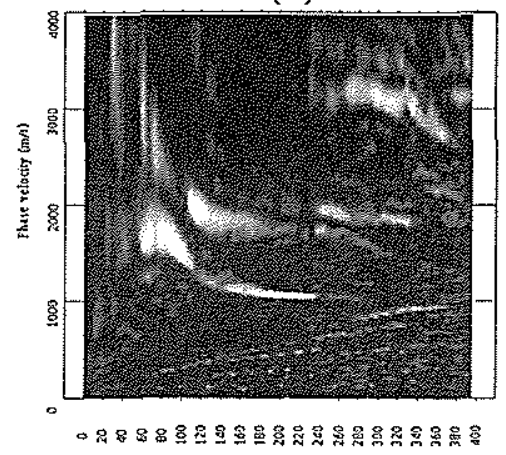

(d)

Figure 6. Vertical component of particle velocities and its phase-velocity images for a model with $5 \mathrm{~mm}$ thickness first layer. (a) Waveforms and (b) a phase-velocity image for $100 \mathrm{KHz}$ pulse. (c) Waveforms and (d) a phase-velocity image for $500 \mathrm{KHz}$ pulse.

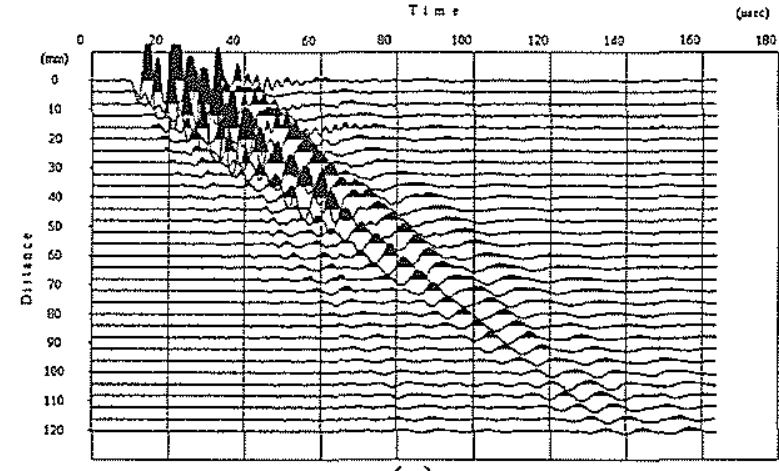

(a)

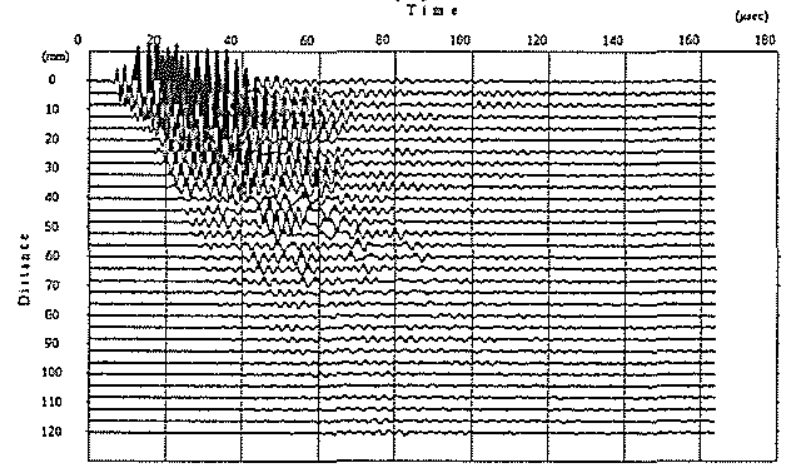

(c)

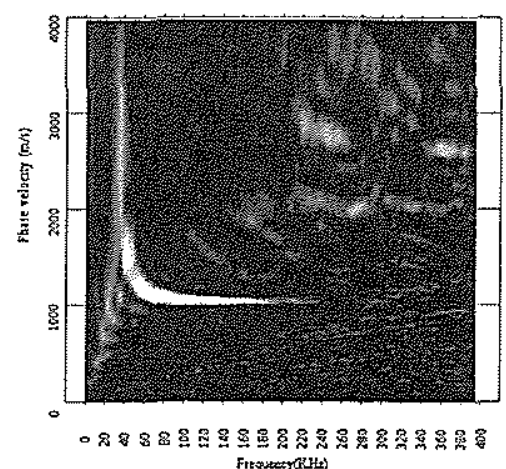

(b)

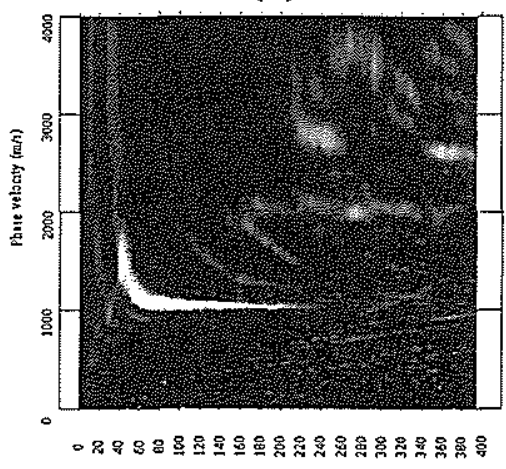

(d)

Figure 7. Vertical component of particle velocities and its phase-velocity images for a model with $10 \mathrm{~mm}$ thickness first layer. (a) Waveforms and (b) a phase-velocity image for $100 \mathrm{KHz}$ pulse. (c) Waveforms and (d) a phase-velocity image for $500 \mathrm{KHz}$ pulse. 


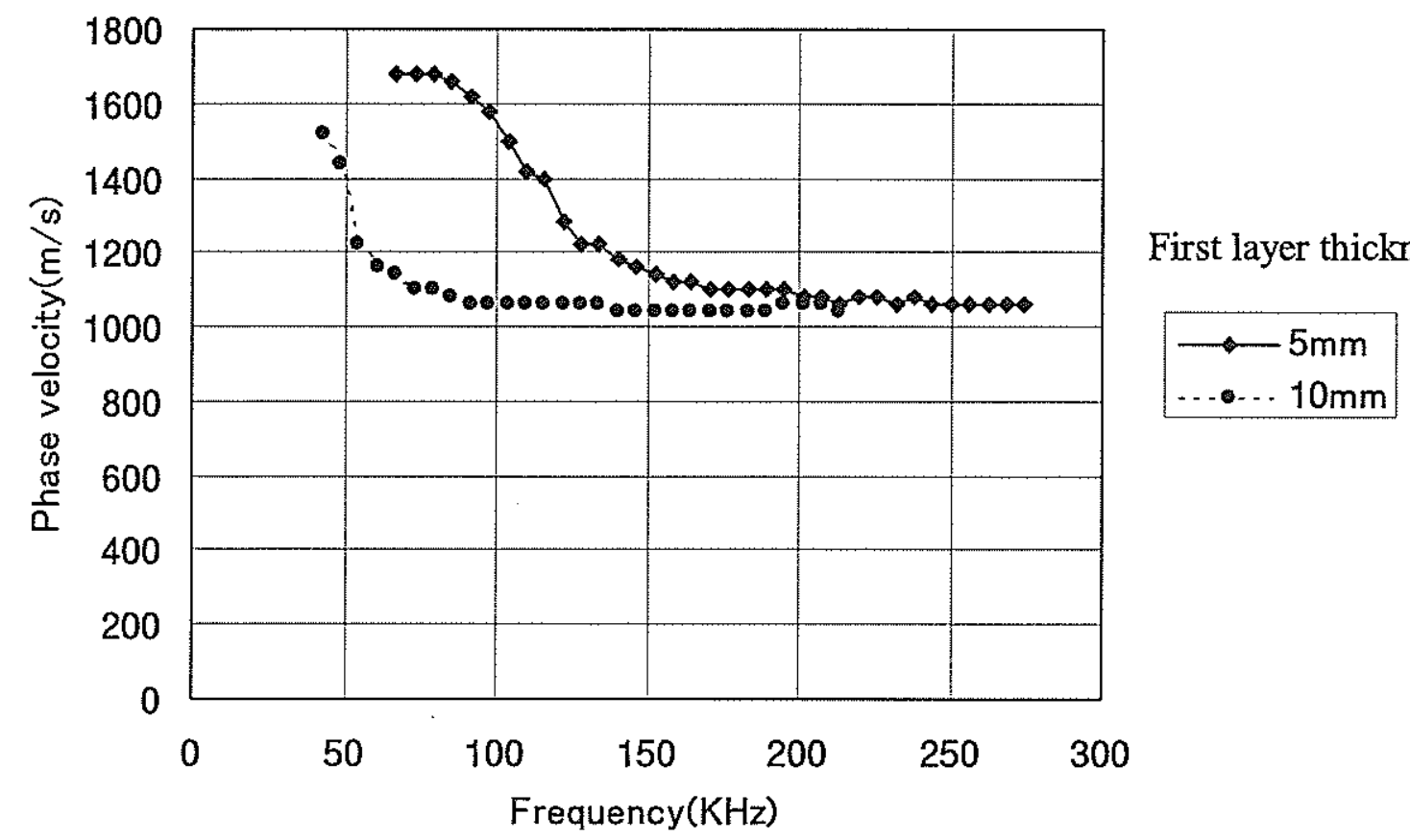

Figure 8. Dispersion curves for the two-layer models. Phase velocities are picked as the maximum amplitude in each frequency on the phase velocity images.

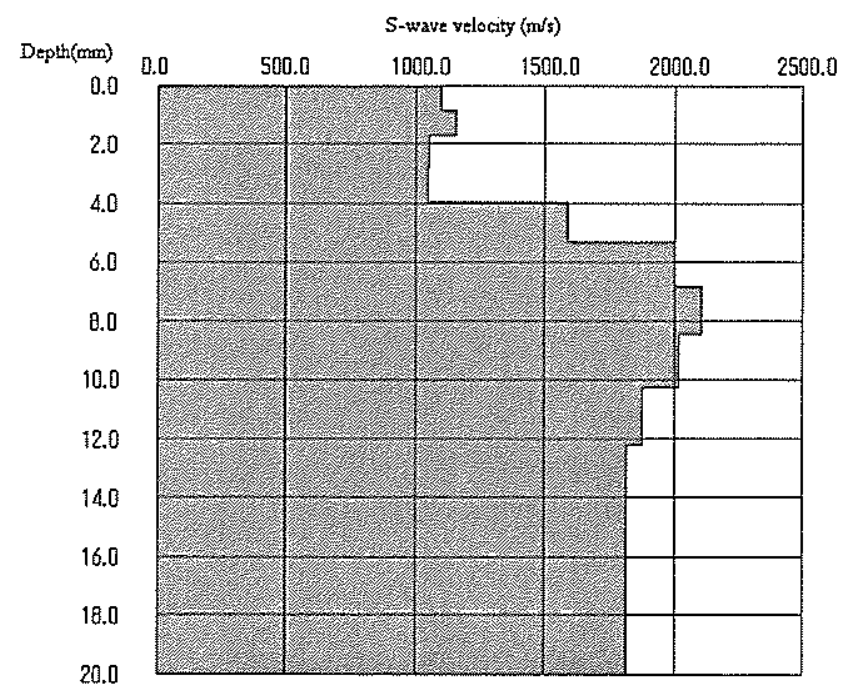

(a)

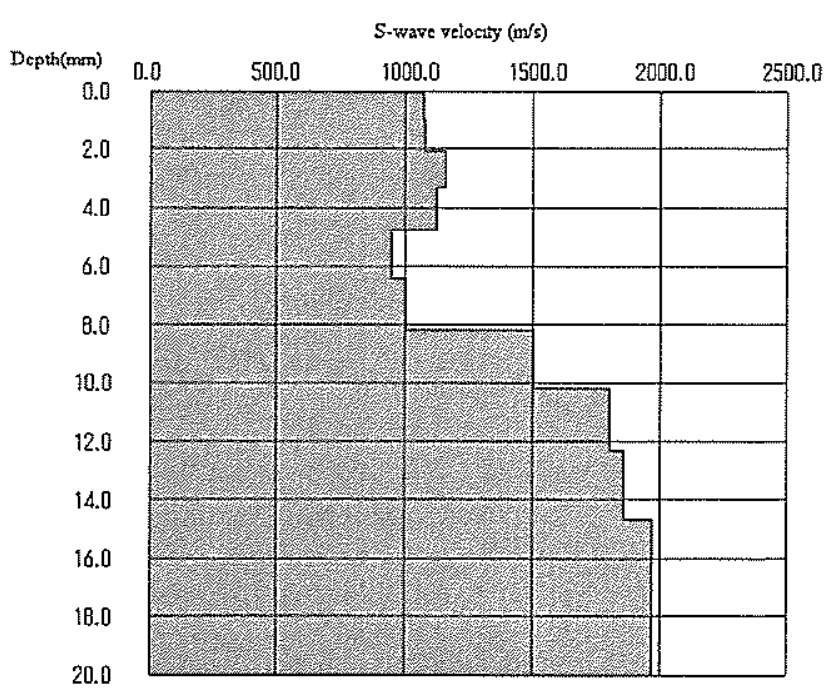

(b)

Figure 9. Inverted velocity models (S-wave velocity) for the two-layer models. (a) A inverted model for a model with $5 \mathrm{~mm}$ thickness first layer. (b) A inverted model for a model with $10 \mathrm{~mm}$ thickness first layer. 